\title{
Biosynthesis of Spinosyn A
}

\section{Key words}

spinosyn A

biosynthesis

Diels-Alder reaction

Rauhut-Currier

reaction

\section{SYNFACTheh}

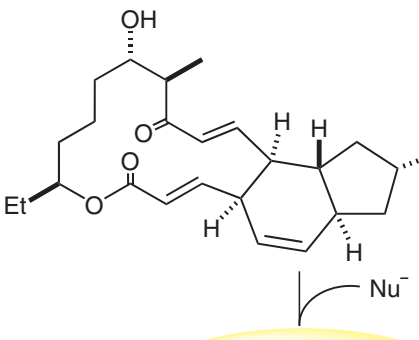

SpnL-mediated Rauhut-Currier reaction

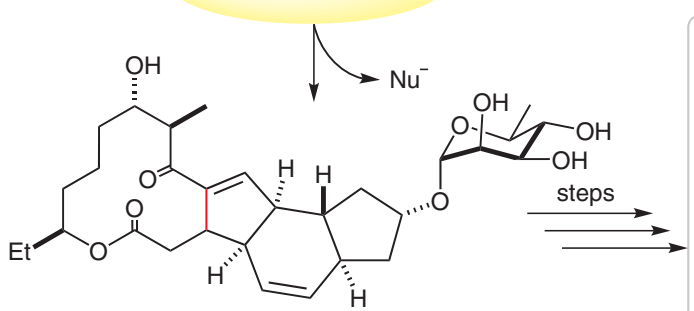
$\mathrm{H}$

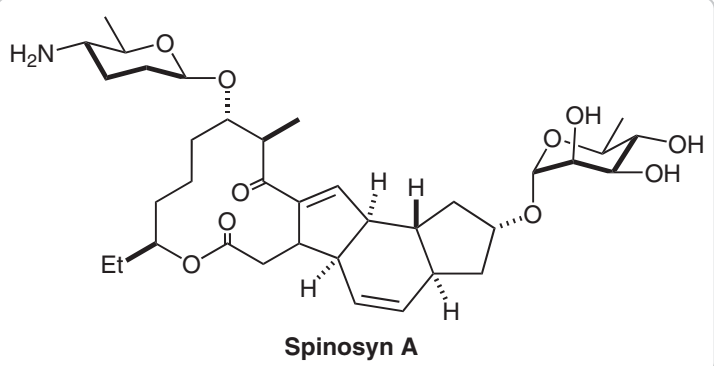

Significance: The authors report a detailed study on the biosynthesis of spinosyn $\mathrm{A}$, an important insecticide from the bacterium Saccharopolyspora spinosa. By systematically investigating the enzymes from the spinosyn A biosynthetic gene cluster overexpressed from Escherichia coli, two important cyclases were identified. The roles of these two enzymes, SpnF and SpnL, are the catalysis of a Diels-Alder reaction and a RauhutCurrier cyclization, respectively. The Diels-Alder reaction was shown to undergo a 500-fold rate enhancement compared to the non-catalyzed reaction.
Comment: Many cyclohexene-containing secondary metabolites are proposed to be biosynthesized via Diels-Alder-type key steps. However, only a few isolated enzymes are known to be a part of biosynthetic pathways where this reaction is presumed to be operative. The present study reveals for the first time, that an enzyme with solely this role exists. As such the SpnF, as a part of the spinosyn biosynthesis, can be considered as a true Diels-Alderase.

SYNFACTS Contributors: Benjamin List, Lars Ratjen 